\title{
Stroke Angel: Effect of Telemedical Prenotification on In-Hospital Delays and Systemic Thrombolysis in Acute Stroke Patients
}

\author{
Patrick Andreas Eder ${ }^{a, b}$ Gunter Laux ${ }^{b}$ Asarnusch Rashid ${ }^{a}$ Tobias Kniess $^{c}$ \\ Karl Georg Haeusler ${ }^{d}$ Layal Shammas ${ }^{a}$ Bernd Griewing ${ }^{e}$ Susanne Hofmann ${ }^{f}$ \\ Stephanie Stanglg Silke Wiedmann ${ }^{\text {h }}$ Viktoria Rücker ${ }^{g}$ Peter U. Heuschmann ${ }^{g}{ }^{\text {i }}$ \\ Hassan Soda ${ }^{f}$ Stroke Angel Study Group \\ annovation management, Zentrum für Telemedizin Bad Kissingen, Bad Kissingen, Germany; ${ }^{b}$ Department of General \\ Practice and Health Services Research, University Hospital Heidelberg, Heidelberg, Germany; 'Department of \\ Neurological Rehabilitation, Campus Rhön Klinikum AG, Bad Neustadt/Saale, Germany; 'Department of Neurology, \\ University Hospital Würzburg, Würzburg, Germany; 'Medical Board Division, Campus Rhön Klinikum AG, Bad Neustadt/ \\ Saale, Germany; fDepartment of Neurology, Campus Rhön Klinikum AG, Bad Neustadt/Saale, Germany; 9Institute of

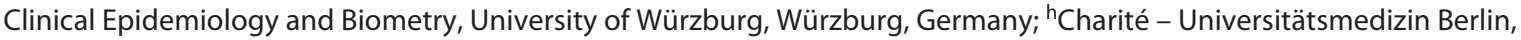 \\ corporate member of Freie Universität Berlin, Humboldt-Universität zu Berlin, and Berlin Institute of Health, Berlin,

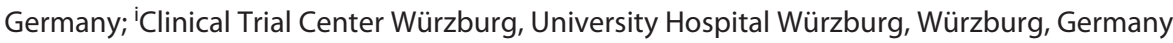

\section{Keywords}

Telemedicine · Acute ischemic stroke · Emergency medical services · Audit · Intravenous thrombolysis

\begin{abstract}
Introduction: Door-to-CT scan time (DCT) and door-to-needle time (DNT) are important process measures in acute ischemic stroke (AIS) patients undergoing intravenous thrombolysis (IVT). We examined the impact of a telemedical prenotification by emergency medical service (EMS) (called the "Stroke Angel" program) on DCT and DNT and IVT rate compared to standard of care. Patients and Methods: Two prospective observational studies including AIS patients admitted via EMS from 2011 to 2013 (cohort l; $n=496$ ) and from January 1, 2015 to May 31, 2018 (cohort II; $n=349$ ) were conducted. After cohort I, the 4-Item Stroke Scale and a digital thrombolysis pro-
\end{abstract}

tocol were added. Multivariable logistic and linear regression analysis was performed. Results: In cohort I, DCT was lower in the intervention group (13 vs. 26 min using standard of care; $p<0.001$ ), but no significant difference in median DNT (35 vs. 39 min; $p=0.24$ ) was observed. In cohort II, a reduction of DCT ( 8 vs. $15 \mathrm{~min} ; p<0.001$ ) and DNT ( 25 vs. $29 \min p=0.003$ ) was observed in the intervention group. Compared to standard of care, the likelihood of DCT $\leq 10 \mathrm{~min}$ or DNT $\leq 20 \mathrm{~min}$ in the intervention group was 2.7 (adjusted odds ratio [aOR] 2.7; 95\% Cl: 2.1-3.5) and 1.8 (aOR 1.8; 95\% Cl: 1.1-2.9), respectively. In cohort II, IVT rate was higher (aOR 1.4; 95\% Cl: 1.1-1.9) in the intervention group. Conclusion: Although the positive effects of Stroke Angel in AIS provided a rationale for implementation in routine care, larger studies of practice implementation will be needed. Using Stroke Angel in the prehospital management of AIS impacts on important process measures of IVT delivery.

$\begin{aligned} & \text { karger@karger.com } \\ & \text { www.karger.com/ced }\end{aligned}$
Karger ${ }^{\prime /}$

Patrick Andreas Eder

Innovation Management

Zentrum für Telemedizin Bad Kissingen

Sieboldstraße 7, DE-97688 Bad Kissingen (Germany)

eder@ztm.de 


\section{Introduction}

Clinical evidence underlines the importance of early initiation of systemic thrombolysis for improving outcomes in patients with acute ischemic stroke (AIS) [1]. International guidelines recommend that in-hospital time delay should be kept as low as possible and address the importance of prenotification and stroke scale utilization in the setting of AIS [2-4]. The current German guideline for the treatment of AIS recommend the time from arrival at the hospital to cranial CT (door-to-CT scan time; DCT) $\leq 25 \mathrm{~min}$ and $<60 \mathrm{~min}$ to the start of thrombolytic treatment (door-to-needle time; DNT) [5]. However, in clinical routine, these targets are often not met [6].

A meta-analysis of prospective and retrospective single-center observational studies showed an impact of verbal prenotification of hospital stroke team staff (emergency room nurse or neurologist) on shortening DCT and DNT as well as increasing intravenous thrombolysis (IVT) delivery [7]. Using a similar approach with emergency medical service (EMS) prenotification, stroke scale utilization, and performance feedback to paramedics, the Paramedic Acute Stroke Treatment Assessment (PASTA) trial showed a nonsignificant trend to lower IVT rates and no reduction of DNT in intervention hospitals [8].

Various strategies currently exist to reduce in-hospital time delays (e.g., transferring patients directly from an ambulance to brain imaging by bypassing the emergency department). There has been increasing attention on the effects of telehealth technology in EMS on in-hospital process and outcomes in acute stroke care [9]. Some studies have demonstrated the feasibility of stroke prenotification transferring structured patient information via mobile health applications (telemedical prenotification) [10]. A scoping review by Lumley et al. [11] emphasizes that insufficient evidence precludes recommendations about routine use of such technologies in the prehospital setting of suspected strokes. The purpose of our study was to examine the impact of telemedical prenotification of hospital stroke team staff (emergency room nurse and neurologist), a program called "Stroke Angel," using telemetry to transmit prehospital data on in-hospital time targets compared to standard of care (prenotification via a mobile phone or single call activation).

\section{Materials and Methods}

This is a single-center observational study of 2 cohorts using prospectively collected data on consecutive patients with suspected AIS admitted to the comprehensive stroke unit in Bad Neustadt,
Bavaria, Germany. The data that support the findings of this study are available from the corresponding author on reasonable request.

\section{Setting and Participants}

The Campus Bad Neustadt is located in North-West Bavaria, Germany, a rural area with about 300,000 inhabitants. There are about 900 admissions for AIS every year. The certified comprehensive stroke unit, according to the German Stroke Society, cooperates with the regional EMS (Bavarian Red Cross). The primary task of EMS is averting life-threatening danger and other severe harms to health including stabilization of vital signs and transporting the patients to the hospital. All emergency medical vehicles are tactically led by dispatch centers and forwarded to the patient as required, with the emergency vehicles meeting directly on scene. Overall, 12 ambulances for paramedics and 4 emergency physician vehicles are available in the catchment area, providing EMS transport for over 60,000 cases per year.

\section{The Stroke Angel Initiative}

The Stroke Angel Initiative is an interdisciplinary project which aims to improve acute stroke management using mobile technologies (handheld computer) for decision-making, documentation, and communication support between EMS and in-hospital stroke staff. The primary goal was to define the pre- and intrahospital process of the AIS patients and to reduce system delays in routine care (shown in online suppl. Table 1; for all online suppl. material, see www.karger.com/doi/10.1159/0005514563). According to our experience during 2005 and 2008, the technological robustness (telecommunication software and hardware) of the Stroke Angel system was proven, and information entered was transmitted fully and correctly. Due to technological advances and amendments of the stroke guideline, we tailored the software and hardware to the requirements of the EMS and emergency department iteratively [12].

Within the scope of the Stroke Angel Initiative, process vulnerabilities were identified and then discussed with the local quality team authorized by EMS and hospital executive leadership and consisting of neurologist, nurses, IT specialists, and paramedics, which after alteration led to a significant acceleration. At the regional level, paramedics and in-hospital stroke staff were supported by the quality team to conduct annual quality improvement cycles (plan-do-study-act; PDSA) and annual feedback meetings (called "Angel Workshop"). In 2010, this interdisciplinary team again performed a comprehensive review of the stroke alert workflow [13]. Between January 1, 2011, and December 31, 2013, we prospectively measured time stamps and process data based on results of PDSA cycles by the members of the Stroke Angel Initiative (cohort I).

The data measurement and workflow underwent a redesign through implementation of a stroke-specific digital thrombolysis protocol during hospital care, and the stroke scale (3-Item Stroke Scale; 3I-SS) was modified in 2014, precluding appropriate calculations of quality indicators and to avoid manual data collection. The modified scale is based on the 3-Item Stroke Scale (assessing level of consciousness, gaze, and motor function) and additionally assesses speech disorder, called the 4-Item Stroke Scale (4I-SS) [14]. Between January 1, 2015, and May 31, 2018, we prospectively conducted a second PDSA cycle to re-evaluate the impact of the Stroke Angel program on in-hospital delays and IVT delivery (cohort II), rather than focusing on prehospital workflow and acceptance. 


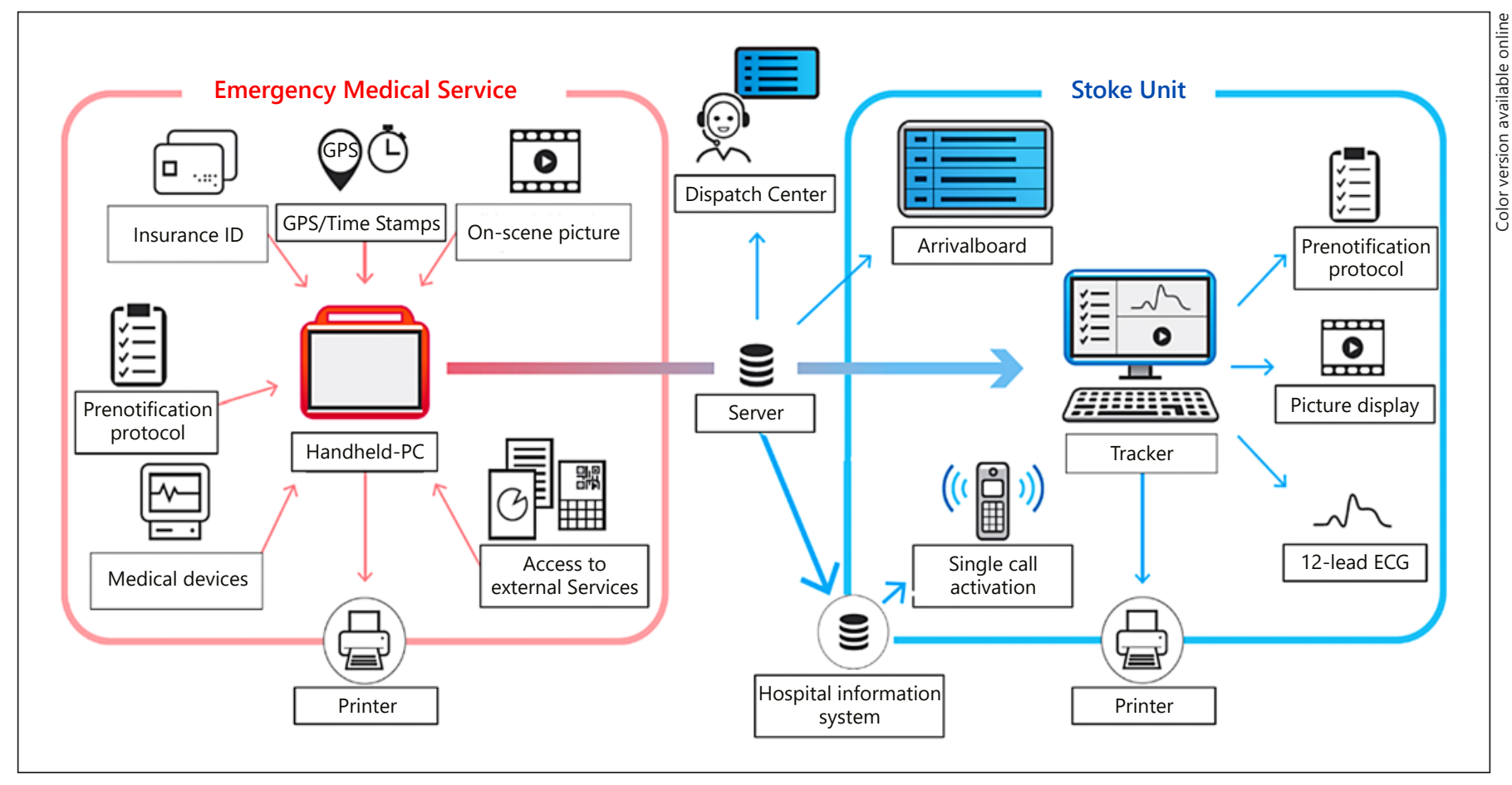

Fig. 1. Technological workflow of the telemedical prenotification system (Stroke Angel) in AIS. AIS, acute ischemic stroke; EMS, emergency medical service; GPS, Global Positioning System; ID, identity; PC, personal computer; ECG, electrocardiogram.

\section{Stroke Angel Intervention}

In 2010, all 12 ambulances were equipped with portable devices (handheld computer called "NIDApad"; medDV, Gießen, Germany) for the purpose of stroke checklists to help the paramedics' examination of patient status; the portable device sends examination findings and patient data wirelessly to an appropriate hospital for prenotification and check-in. Neurologic deficits were graded according to the 4I-SS (shown in online suppl. Table 2). The technical implementation of Stroke Angel was similar in both cohorts as shown in Figure 1. In cohort II, a cross-sectoral platform (digital thrombolysis protocol) was developed that electronically integrates the Stroke Angel data into the hospital's electronic health records. In both cohorts, control cases for brain imaging and IVT delivery consisted of consecutive patients with standard of care defined as prenotification via a mobile phone or single call activation from the same catchment area. In cohorts I and II, EMS can discuss atypical symptoms $24 / 7$ on the mobile phone with the hospital stroke neurologist on duty. Due to statutory requirements, the dispatch center prealerts the stroke unit via a telephone in case of suspected stroke.

\section{Data Collection and Outcome Measures}

Cohorts I and II enrolled patients 18 years or older with suspected AIS admission diagnosis. Patients were further excluded from the analysis in both cohorts if they had no confirmed ischemic stroke (ICD-10 I63) according to discharge diagnosis, and patients with a symptom onset to admission of $>4.5 \mathrm{~h}$ and secondary transport mode as well as implausible or missing metrics were also excluded.
In cohort I, we measured the scene-to-imaging time by manual data extraction from EMS protocols and hospital records. We further revised scene-to-imaging time in cohort II, as we compared the percentage of achieved predefined quality indicators (DCT and DNT) according to predefined targets stated, German AIS guideline recommendations (AWMF No. 030-046) [4] and best practices in the literature [15]. In particular, we calculated the percentage of patients with DCT $\leq 25$ min (guideline recommendation) and $10 \mathrm{~min}$ (best practice goal) and $\mathrm{DNT} \leq 60 \mathrm{~min}$ (guideline recommendation) and 20 min (best practice goal), respectively.

\section{Statistical Methods}

Patients were stratified into 2 groups based on the composite use (by staff) of Stroke Angel defined as the on-scene stroke scale utilization and telemedical prenotification ("all or none"). Summary statistics were expressed as frequencies and proportions for categorical parameters and median values and interquartile range (IQR) for continuous data. One-way ANOVA or the Kruskal-Wallis test ( $>2$ groups) in the case of nonnormally distributed continuous variables was applied. Categorical variables were compared using Pearson's $\chi^{2}$ test.

In cohort I, logistic regression analyses were applied to assess the association of sociodemographic parameters, factors related to disease, and admission with the dichotomous outcome variable (Stroke Angel: yes vs. no). In addition, generalized linear models were applied to evaluate to effect of Stroke Angel and other parameters (i.e. age and ways of admission) on scene-to-imaging time.

In cohort II, we conducted a multivariable logistic regression analysis modeling the association with Stroke Angel and in-hospi- 
Table 1. Clinical characteristics of AIS patients from cohort I stratified by Stroke Angel $(n=496)$

\begin{tabular}{|c|c|c|c|}
\hline & $\begin{array}{l}\text { Stroke Angel group } \\
n=250\end{array}$ & $\begin{array}{l}\text { Control group } \\
n=246\end{array}$ & $p$ value \\
\hline \multicolumn{4}{|l|}{ Age, $n(\%)$} \\
\hline$<55$ & $21(8.4)$ & $16(6.5)$ & \multirow{5}{*}{0.11} \\
\hline $55-64$ & $32(12.8)$ & $38(15.4)$ & \\
\hline $65-74$ & $71(28.4)$ & $48(19.5)$ & \\
\hline $75-84$ & $77(30.8)$ & $95(38.6)$ & \\
\hline$>84$ & $49(19.6)$ & $49(19.9)$ & \\
\hline Female sex, $n(\%)$ & $112(45.0)$ & $110(44.9)$ & 0.99 \\
\hline \multicolumn{4}{|l|}{ NIHSS on admission, $n(\%)$} \\
\hline $0-4$ & $124(49.6)$ & $132(53.7)$ & \multirow{3}{*}{0.59} \\
\hline $5-15$ & $110(44.0)$ & $97(39.4)$ & \\
\hline$>15$ & $16(6.4)$ & $17(6.9)$ & \\
\hline \multicolumn{4}{|l|}{ Way of admission, $n(\%)$} \\
\hline Paramedics & $214(85.6)$ & $55(22.4)$ & \multirow{3}{*}{$<0.001$} \\
\hline Paramedics and emergency physician (EMS) & $31(12.4)$ & $119(48.4)$ & \\
\hline Via general practitioner with EMS & $5(2.0)$ & $72(29.3)$ & \\
\hline \multicolumn{4}{|l|}{ Admission diagnosis, ICD-10, $n(\%)$} \\
\hline I61. Intracerebral hemorrhage & $1(0.4)$ & $2(0.8)$ & \multirow{4}{*}{0.44} \\
\hline I63. Cerebral infarction & $228(91.6)$ & $231(93.9)$ & \\
\hline G45. TIA & $16(6.4)$ & $12(4.9)$ & \\
\hline Others & $4(1.6)$ & $1(0.4)$ & \\
\hline Admission during weekend, $n(\%)$ & $75(30.0)$ & $73(29.7)$ & 0.94 \\
\hline Admission during out-of-hour-time, $n(\%)$ & $152(60.8)$ & $146(59.3)$ & 0.74 \\
\hline \multicolumn{4}{|l|}{ Years on admission, $n(\%)$} \\
\hline 2011 & $84(33.6)$ & $100(40.7)$ & \multirow{3}{*}{0.12} \\
\hline 2012 & $103(41.2)$ & $80(32.5)$ & \\
\hline 2013 & $63(25.2)$ & $66(26.8)$ & \\
\hline \multicolumn{4}{|l|}{ Distance from stroke location to hospital, $n(\%), \mathrm{km}$} \\
\hline$\leq 5 \mathrm{~km}$ & $37(14.8)$ & $30(12.2)$ & \multirow{5}{*}{0.64} \\
\hline$<5$ to $\leq 10 \mathrm{~km}$ & $45(18.0)$ & $35(14.2)$ & \\
\hline$>10$ to $\leq 15 \mathrm{~km}$ & $40(16.0)$ & $43(17.5)$ & \\
\hline$>15$ to $\leq 20 \mathrm{~km}$ & $82(32.8)$ & $86(35.0)$ & \\
\hline$>20 \mathrm{~km}$ & $46(18.4)$ & $52(21.1)$ & \\
\hline Accuracy of estimated arrival time, median (IQR) ${ }^{\mathrm{a}}$ & $0.0([-3]-4)$ & na & na \\
\hline Time at scene, median (IQR), min & $18.0(15-24)$ & $18.5(14-25)$ & 0.86 \\
\hline Transportation time, median (IQR), min & $20.5(13-27)$ & $23.0(17-32)$ & 0.01 \\
\hline \multicolumn{4}{|l|}{ IVT delivery, $n(\%)$} \\
\hline Yes & $100(40.0)$ & $70(28.5)$ & \multirow{2}{*}{0.01} \\
\hline No & $150(60.0)$ & $176(71.5)$ & \\
\hline DCT, median (IQR), min & $13.0(9-19)$ & $26.0(16-38)$ & $<0.001$ \\
\hline Scene-to-imaging time, median (IQR), min & $54.0(46-65)$ & $70.5(58-85)$ & $<0.001$ \\
\hline DNT, median (IQR), min & $35.0(27-46)$ & $39.0(28-49)$ & 0.24 \\
\hline \multicolumn{4}{|l|}{ mRS (before index stroke), $n(\%)$} \\
\hline $0-2$ & $223(89.6)$ & $207(84.5)$ & \multirow[b]{2}{*}{0.09} \\
\hline $3-5$ & $26(10.4)$ & $38(15.5)$ & \\
\hline
\end{tabular}

AIS, acute ischemic stroke; EMS, emergency medical service; NIHSS, National Institute Health Stroke Scale; DCT, door-to-CT scan time; DNT, door-to-needle time; mRS, modified Rankin Scale; na, not applicable; IVT, intravenous thrombolysis. ${ }^{a}$ Analyses were restricted to patients without missing values. Data were from cohort I (2011-2013): patients transported by EMS, with ischemic stroke and IVT delivery. 
tal delay and systemic thrombolysis (Stroke Angel with score: yes vs. no score). The aim was to evaluate the importance of stroke scale telemetry. Relationships were further examined and fitted for each outcome with data from quality cohort II with Stroke Angel as the only covariate in each model (unadjusted model) and with statistically significant interactions $(p<0.1)$ from the additional covariates and the unadjusted model (forward selection method). We added the attendance of an emergency physician to the regression model to eliminate therapeutic differences on-scene such as antihypertensive therapy or intravenous access of EMS according to local clinical practice recommendations. We conducted a subgroup analysis in cohort II, restricting suspected and confirmed stroke patients to NIHSS $\leq 4$ on admission (shown in online suppl. material) [16].

The Hosmer-Lemeshow test statistic was used for assessing the goodness of fit (calibration) and Nagelkerkes pseudo R-square for estimating explained variance. The results of both cohorts are expressed as odds ratio (OR) with $95 \%$ confidence intervals (95\% CI) using exponentiation from the regression parameter estimates. The results of the multivariable logistic regression models should be interpreted cautiously as associations may be significant by chance. All $p$ values were 2 -tailed, and $<5 \%$ were considered as significant. All analyses were performed using Statistical Package for the Social Sciences Statistics Version 25 (IBM Corp., Armonk, NY, USA).

\section{Results}

\section{Patients' Characteristics of Cohort I}

Of 3,064 patients admitted with suspected AIS, 903 (29.5\%) patients fulfilled the inclusion criteria. Of those, 496 (16.2\%) patients were transported via EMS and eligible for our analysis; Stroke Angel was used in 250 (50.4\%) of these cohort I patients (shown in online suppl. Fig. 1). There were no significant differences regarding patientrelated factors or time of hospital admission (shown in Table 1). In the Stroke Angel cohort, there was a shorter time of transportation (20.5 min [IQR 13-27] vs. $23.0 \mathrm{~min}$ [IQR 17-32]; $p=0.01$ ) and a higher rate of IVT delivery ( 40.0 vs. $28.5 \%$; $p=0.01$ ). The way of admission (paramedics, paramedics and emergency physician, or via general practitioner with EMS) differed between the Stroke Angel and control groups $(p<0.001)$. There were no differences in the accuracy of arrival time estimated by EMS and time at scene. The distribution of socioeconomic and admission-related factors (i.e., way of admission and mRS before the index stroke) of individuals stratified by Stroke Angel is shown in Table 1 (cohort I).

\section{Probability of Using Stroke Angel in Cohort I}

We performed logistic regression analysis to investigate factors associated with the probability of using Stroke Angel (shown in online suppl. Table 3). In univariable models, a significant association was only revealed between Stroke Angel and way of admission. Here, the strongest association for Stroke Angel was seen for admission by paramedics (OR 15.5; 95\% CI: 9.4-25.6) compared to admission by paramedic and emergency physician (reference). Moreover, hospitalization via a general practitioner with EMS reduced the chance of using Stroke Angel (OR 0.3; 95\% CI: 0.1-0.7). In the fully adjusted model, admission by paramedics was still strongly associated with Stroke Angel use (adjusted Odds Ratio [aOR] 32.2; 95\% CI: 16.7-62.2). Furthermore, utilizing Stroke Angel was more likely in 2012 compared to 2011 (OR 1.5; 95\% CI: 1.0-2.3) but not in 2013 (OR 1.1; 95\% CI: 0.7-1.8) compared to 2011 (reference). In multivariable analysis, the probability of using Stroke Angel between 2011 and 2013 got stronger and statistically significant (2012: aOR 2.5, 95\% CI: 1.4-4.6; 2013: aOR 5.2, 95\% CI: 2.5-10.9).

\section{Scene-to-Imaging Time in Cohort I}

In univariable linear regression analysis, we observed that Stroke Angel reduced scene-to-imaging time about 15 min compared to standard care $(\beta-15.7 ; 95 \%$ CI: $-19.3,-12.2)$. This effect remained stable after adjustment for Stroke Angel use, way of admission, age, sex, NIHSS (at the beginning of acute phase), time of admission, day of admission, distance to hospital, year of admission, and mRS (before the index stroke). Some other variables reached significance in univariable modeling (i.e., day and way of admission and age) but not in the full model. All results of linear regression are shown in online suppl. Table 4.

\section{Patients' Characteristics of Cohort II}

Between 2015 and 2018, 2,923 patients were admitted. Of those, 1,124 (38.5\%) patients fulfilled the inclusion criteria, in which $15.6 \%(n=455)$ used Stroke Angel, whereas $22.9 \%(n=669)$ did not. The final analysis included $n=1,124$ patients, of whom $356(12.2 \%)$ had a confirmed ischemic stroke at discharge and received IVT (shown in Table 2). Thus, 349 (11.9\%) patients were identified as eligible (shown in online suppl. Fig. 2).

\section{DCT and DNT in Cohort II}

Univariate analysis demonstrated that Stroke Angel was associated with greater likelihood of performing cranial CT $\leq 25$ and $10 \mathrm{~min}$, as well as receiving IVT delivery within 60 and 20 min after ED arrival (shown in Table 3). In multivariable analysis, the likelihood of DCT $\leq 25 \mathrm{~min}$ was 4.0 (95\% CI: 2.8-5.9) for Stroke Angel use. After adjusting for symptom onset (calculated by EMS on-scene) 
Table 2. Clinical characteristics of the suspected ischemic stroke cohort for the study group Stroke Angel versus the control group from cohort II $(n=1,124)$

\begin{tabular}{|c|c|c|c|}
\hline & $\begin{array}{l}\text { Stroke Angel group } \\
n=455\end{array}$ & $\begin{array}{l}\text { Control group } \\
n=669\end{array}$ & $p$ value \\
\hline Age, median (IQR) & $78.8(70.3-85.1)$ & $78.5(68.8-85.2)$ & 0.32 \\
\hline \multicolumn{4}{|l|}{ Age groups, $n(\%)$} \\
\hline$<55$ & $26(5.7)$ & $54(8.1)$ & \multirow{5}{*}{0.21} \\
\hline $55-64$ & $44(9.7)$ & $81(12.1)$ & \\
\hline $65-74$ & $87(19.1)$ & $111(16.6)$ & \\
\hline $75-84$ & $184(40.4)$ & $245(36.6)$ & \\
\hline$>84$ & $114(25.1)$ & $178(26.6)$ & \\
\hline \multicolumn{4}{|l|}{ Sex, $n(\%)$} \\
\hline Male & $226(49.7)$ & $311(46.5)$ & \multirow{2}{*}{0.29} \\
\hline Female & $229(50.4)$ & $358(53.5)$ & \\
\hline NIHSS on admission, median (IQR) & $6(3-11)$ & $5(2-10)$ & 0.001 \\
\hline \multicolumn{4}{|l|}{ NIHSS on admission, $n(\%)$} \\
\hline $0-4$ & $198(41.5)$ & $324(48.4)$ & \multirow{5}{*}{0.03} \\
\hline $5-10$ & $143(31.4)$ & $192(28.7)$ & \\
\hline $11-16$ & $64(14.1)$ & $63(9.8)$ & \\
\hline $16-21$ & $42(9.2)$ & $56(8.8)$ & \\
\hline$\geq 22$ & $17(3.7)$ & $34(5.1)$ & \\
\hline mRS (before index stroke), median (IQR) & $3(2-4)$ & $2(2-4)$ & 0.006 \\
\hline Barthel index on admission, median (IQR) & $50(20-75)$ & $60(25-80)$ & 0.002 \\
\hline \multicolumn{4}{|l|}{ Way of admission, $n(\%)$} \\
\hline Paramedic & $146(32.1)$ & $158(27.0)$ & \multirow{4}{*}{$<0.001$} \\
\hline Paramedic and emergency physician & $265(58.2)$ & $324(52.4)$ & \\
\hline Helicopter & $0(0.0)$ & $6(0.5)$ & \\
\hline General practitioner and EMS & $44(9.7)$ & $181(27.1)$ & \\
\hline Length of in-hospital stay, median (IQR), days & $9(7-11)$ & $8(6-11)$ & 0.04 \\
\hline DNT, median (IQR), min & $25(20-35)$ & $29(23-40)$ & 0.003 \\
\hline DCT, median (IQR), min & $8(5-14)$ & $15(7-28)$ & $<0.001$ \\
\hline Symptom onset-to-door time, median (IQR), min & $127.5(75-350)$ & $178.3(90-440)$ & $<0.001$ \\
\hline Mortality, $n(\%)$ & $15(3.3)$ & $18(2.7)$ & 0.59 \\
\hline
\end{tabular}

EMS, emergency medical service; IQR, interquartile range; NIHSS, National Institute Health Stroke Scale; mRS, modified Rankin Scale; DNT, door-to-needle-time; DCT, door-to-CT scan time.

Table 3. Results of the multivariable logistic regression analysis of cohort II associated with quality outcomes $(n=1,124)$

\begin{tabular}{lllll}
\hline Outcome & $\begin{array}{l}\text { Stroke Angel group } \\
\%(n / N)\end{array}$ & $\begin{array}{l}\text { Control group } \\
\%(n / N)\end{array}$ & cOR $(95 \% \mathrm{CI})$ & aOR (95\% CI) \\
\hline $\begin{array}{l}\text { Suspected stroke } \\
\text { DCT } \leq 25 \text { min }\end{array}$ & $91.9(418 / 455)$ & $72.5(485 / 669)$ & $4.98(3.43-7.23)^{\dagger}$ & $4.02(2.75-5.88)^{\dagger}$ \\
$\quad$ DCT $\leq 10$ min & $63.4(288 / 455)$ & $38.0(254 / 669)$ & $2.82(2.20-3.60)^{\dagger}$ & $2.70(2.10-3.47)^{\dagger}$ \\
$\quad$ IVT delivery & $38.0(173 / 455)$ & $27.4(183 / 669)$ & $1.63(1.26-2.10)^{\dagger}$ & $1.44(1.07-1.92)^{*}$ \\
Confirmed ischemic stroke & & & & \\
$\quad$ DNT $\leq 60$ min & $96.4(161 / 167)$ & $90.1(164 / 182)$ & $2.94(1.14-7.61)^{\dagger}$ & $3.15(1.31-7.16)^{*}$ \\
DNT $\leq 20$ min & $35.3(59 / 167)$ & $23.1(42 / 182)$ & $1.82(1.14-2.91)^{*}$ & $1.83(1.14-2.94)^{*}$ \\
\hline
\end{tabular}

DCT, door-to-CT scan time; DNT, door-to-needle time; IVT delivery, intravenous thrombolysis via tissue plasminogen activator; cOR, crude odds ratio; aOR, adjusted odds ratio. Adjustment: symptom onset, NIHSS on admission, emergency physician attendance, age, and gender. The reference in the logistic model was defined as the negative value of each dichotomous variable. ${ }^{\dagger} p<0.001 .{ }^{*} p<0.05$.

Telemedical Prenotification in Acute Stroke 
and NIHSS on admission (calculated by neurologist), there was a 2.7 (95\% CI: 2.1-3.5) higher chance for DCT within $10 \mathrm{~min}$ in the Stroke Angel group. After adjustment for symptom onset and NIHSS, Stroke Angel increased the likelihood of IVT delivery compared to standard of care. IVT delivery occurred in 173 (38.0\%) of 455 patients versus $183(27.4 \%)$ of 669 assigned controls (aOR 1.4; 95\% CI: 1.0-1.9). There was a significant difference between patients in the Stroke Angel group and standard of care with the OR of 1.8 (95\% CI: 1.1-2.9) for DNT $\leq 20$ min (adjusted for emergency physician attendance and symptom onset). We had no evidence of multicollinearity. Calibration and variance results from multivariable regression models are shown in online suppl. Table 5.

\section{Discussion}

In 2 prospective cohort studies, we found that the Stroke Angel program for patients with suspected AIS is feasible and significantly shortens the DCT and, after workflow modification in cohort II, also DNT. Thus, Stroke Angel improves the odds of systemic thrombolytic therapy use as compared with a conventional prenotification workflow protocol. We also identified way and year of admission as determinants associated with acceptance in using telemedical prenotification.

Analysis revealed that the presence of an emergency physician on admission had a negative impact on Stroke Angel utilization. In the catchment area, emergency physicians maintained the standard of care with telephone prenotification directly with the neurologist on duty, since the emergency physicians' vehicles were not equipped with the Stroke Angel system. Although we were able to show in our analysis that the time at scene is not extended, the restrained use and acceptance seems to be due to the use of telemedicine which creates a subjective feeling of prolonged stay on site. Several determinants may be implicated in inaccurate or missing telemedical prenotification. This may include the experience of the EMS personnel, knowledge about the in-hospital effect, clinical inertia, subjective feel of futility, short distance to the receiving hospital, and technical challenges.

The unexpected result of an association between determinants using telemedical prenotification and in-hospital delays has not yet been adequately reported. Even though the Stroke Angel Initiative showed a reduction of in-hospital delays and increased IVT delivery over time, we found that EMS did not confer greater likelihood of telemedical prenotification, indicating missed opportunities to avoid in-hospital delays compared to telephone prenotification. This may be due to the lack of guideline implementation and evidence supporting the implementation of Stroke Angel. Although the positive effects of telemedical prenotification in AIS provided a rationale for implementation in routine care, larger studies of practice implementation will be needed to determine whether reduction of in-hospital delays by telemedical prenotification leads to improved outcomes. Thus, our study implicated that time-consuming telephone calls can be limited (narrowed) to particular situations. Implementation of the Stroke Angel system also involved various costs for hardware, installation, configuration, and maintenance/ support per hospital about 10,000 EUR p.a. and 5,000 EUR per ambulance (conservative assumption).

Andrew et al. [17] showed that using telemedical prenotification via mobile smart phone application (Stop Stroke; Pulsara, Inc., Bozeman, MT, USA) is associated with achieving DNT <60 min (OR 1.8; 95\% CI: 1.1-3.0). Over $35 \%$ of patients in our study who were in the Stroke Angel group received systemic thrombolysis within 20 min after arrival. With multiple concurrent strategies, Meretroja et al. [18] cut the median DNT to $20 \mathrm{~min}$ in 2011. A pilot study (med-on-@ix) assessing telemedicine feasibility in prehospital AIS care in Aachen, Germany, used a 14-item stroke history checklist during teleconsultation to support EMS personnel. The stroke checklist was then sent via fax and handed over to the neurologist on duty. Prenotification was conducted via a cell phone, and no data transmission was performed. Bergrath et al. [19] showed that such a system leads to no reduction in DCT and did not improve prehospital diagnostic quality.

To counteract determinants of nonadherence, some stroke networks have introduced the so-called learning collaborative, similar to the Stroke Angel Initiative, which strives to bridge the gap between current and ideal practice [20]. Recent observational and pilot studies show that DCT and DNT have improved during the past decade and implementation strategies such as 'stroke code,' 'stroke bundle,' or 'pit crew' [4]. New strategies for improving AIS care delivery have been implemented such as Mobile Stroke Units with and without telemedicine [21], telemedical consultation of a remote neurologist for onscene support [22], or point-of-care laboratory [23]. These implementation strategies offer the opportunity for safe reorganization and are often bundled into a bouquet of single quality initiatives reducing time to treatment [24]. 


\section{Strength and Limitations}

The results of this study should be interpreted with consideration to the following limitations: first, the study design did not include cluster or individual randomization, and the decision to use Stroke Angel was left to the acceptance by EMS personnel and availability of mobile technology. Thus, it cannot be excluded that some of our findings is biased by confounding. Second, we assume that electronic documentation of quality measures was analogous to actual clinical practice. Despite the fact that data were routinely checked for plausibility, coding errors cannot be ruled out. Third, given the bundled approach in our study, it is not clear whether the contributions of each portion of the bundle were responsible for the improvements or if they were due to the comprehensive nature of the stroke care that patients received. Fourth, attempting to achieve shorter in-hospital delay may lead to rushed assessments, inappropriate patient selection, dosing errors, higher probability of complications, and challenging working conditions. Moreover, data analyses revealed a low Nagelkerkes $R^{2}$, suggesting that there are further factors that influence in-hospital delays. Even with adjustment for each confounding variable by including it as an explanatory variable in a multivariable regression model, there remains some uncertainty due to the disparity between demographic profiles. Finally, this is an observational study not allowing for conclusions on the causality of the associations.

\section{Conclusion}

Telemedical prenotification connects all members of acute stroke care, including paramedics and emergency triage, and allows robust streamlined workflow logistics minimizing delays in assessment, imaging, and treatment initiation. In the future, implementation of telemedical prenotification in routine AIS care merits attention precluding jeopardized patient outcomes. Future work should consider investigation into determinants that perpetuate Stroke Angel nonuse to further accelerate quality improvement strategies.

\section{Acknowledgements}

We acknowledge the sustained and committed contribution of all working group members, especially Detlef Maag for data extraction of the stroke quality collaborative in Bad Neustadt.

\section{Statement of Ethics}

An ethical approval was obtained from the local ethics committee (No. FB VVV 030-B-11/06). Ethical approval for informed consent of AIS patients in cohort II was waived by the Ethics Committee of the Bavarian Chamber of Physicians as no intervention on human subject was performed, and the data collection was completely anonymous (No. 218-111). Data management was approved by the local data protector. Cohort I: informed consent was obtained from the patients for their anonymized information to be published in this article. Cohort II: informed consent was not obtained from the patients for their anonymized information to be published in this article.

\section{Conflict of Interest Statement}

The authors (P.A.E., G.L., A.R., T.K., L.S., B.G., S.H., S.S., V.R., and H.S.) declare that the research was conducted in the absence of any commercial or financial relationships that could be construed as a potential conflict of interest. P.U.H. reports research grants from the German Ministry of Research and Education, German Research Foundation, European Union, Federal Joint Committee (G-BA) within the Innovationfond, Charité, Berlin Chamber of Physicians, German Parkinson Society, University Hospital Würzburg, Robert-Koch-Institute, German Heart Foundation, CharitéUniversitätsmedizin Berlin (within MonDAFIS; MonDAFIS is supported by an unrestricted research grant to the Charité from Bayer), University Göttingen (within FIND-AF-randomized; FIND-AF randomized is supported by an unrestricted research grant to the University Göttingen from Boehringer-Ingelheim), and University Hospital Heidelberg (within RASUNOA-prime; RASUNOA-prime is supported by an unrestricted research grant to the University Hospital Heidelberg from Bayer, BMS, Boehringer-Ingelheim, and Daiichi Sankyo), outside submitted work. S.W. reports grants from the German Federal Ministry of Education and Research and Deutsche Herzstiftung e.V. K.G.H. reports study grants by Bayer Healthcare and honoraria from Bayer Healthcare, Pfizer, Boehringer-Ingelheim, Bristol-Myers Squibb, Daiichi Sankyo, Edwards Lifesciences, Medtronic, and Biotronik.

\section{Funding Sources}

The Stroke Angel Initiative was funded by the German Federal Ministry of Education and Research: research project PerCoMed (Pervasive Computing in of networked medical care - Funding No. 16I1546) and INSPIRE (Improving Service Productivity in Healthcare - Sponsorship No. 01 FL10080). The Stroke Angel Initiative was supported by the German Stroke Assistance, Boehringer-Ingelheim, the medDV GmbH (Gießen, Germany), and the Competence Network Stroke. The authors received no specific funding for the study design, data collection, data analysis, data interpretation, or writing of the report. Members of the Stroke Angel group: Dr. Asarnusch Rashid, Dr. Tobias Kniess, Dr. Ing. Layal Shammas, Prof. Dr. Bernd Griewing, Susanne Hofmann, Dr. Hassan Soda, Patrick Andreas Eder, Uwe Kippnich, Dr. Volker Ziegler, Heiko Stäblein, Stefan-André Ruß, Dr. Erich Hiermann, Carina Hopfengart, and Carsten Rausch.

Cerebrovasc Dis 2021;50:420-428 


\section{Author Contributions}

P.A.E., A.R., S.H., and H.S. had access to the electronic health data registry, and P.A.E., A.R., S.H., S.S., and G.L. had access to the hospital episode statistics data. P.U.H., H.S., A.R., V.R., S.W., and
P.A.E. designed the study, obtained data, performed and interpreted statistical analysis, and drafted the manuscript. K.G.H., P.U.H., V.R., P.A.E., and G.L. interpreted statistical analysis and critically reviewed the manuscript. All authors reviewed and edited the manuscript and approved the final version of the manuscript.

\section{References}

1 Zerna C, Thomalla G, Campbell BCV, Rha $\mathrm{J}-\mathrm{H}$, Hill MD. Current practice and future directions in the diagnosis and acute treatment of ischaemic stroke. Lancet. 2018;392(10154): 1247-56.

2 Man S, Xian Y, Holmes DN, Matsouaka RA, Saver JL, Smith EE, et al. Association between thrombolytic door-to-needle time and 1-Year mortality and readmission in patients with acute ischemic stroke. JAMA. 2020;323(21): 2170-84.

3 Powers WJ, Rabinstein AA, Ackerson T, Adeoye OM, Bambakidis NC, Becker K, et al. Guidelines for the early management of patients with acute ischemic stroke: 2019 update to the 2018 guidelines for the early management of acute ischemic stroke: a guideline for healthcare professionals from the American Heart Association/American Stroke Association. Stroke. 2019;50(12):e344-418.

4 Kobayashi A, Czlonkowska A, Ford GA, Fonseca AC, Luijckx GJ, Korv J, et al. European Academy of Neurology and European Stroke Organization consensus statement and practical guidance for pre-hospital management of stroke. Eur J Neurol. 2018;25(3):425-33.

5 Ringleb P, Hamann G, Röther J, Jansen O, Groden C, Veltkamp R. Akuttherapie des ischämischen Schlaganfalls - Rekanalisierende Therapie. Akt Neurol. 2016;43(2):82-91.

6 Ragoschke-Schumm A, Walter S, Haass A, Balucani C, Lesmeister M, Nasreldein A, et al. Translation of the 'time is brain' concept into clinical practice: focus on prehospital stroke management. Internat. J. Stroke. 2014;9(3): 333-40.

7 Huang Q, Zhang JZ, Xu WD, Wu J. Generalization of the right acute stroke promotive strategies in reducing delays of intravenous thrombolysis for acute ischemic stroke: a meta-analysis. Medicine. 2018;97(25):e11205.

8 Price C, Shaw L, Islam S, Javanbakht M, Watkins A, McMeekin P, et al. Effect of an en- hanced paramedic acute stroke treatment assessment on thrombolysis delivery during emergency stroke care. A cluster randomized clinical trial. JAMA Neurol. 2020:e200611.

9 Winburn AS, Brixey JJ, Langabeer J, Champagne-Langabeer T. A systematic review of prehospital telehealth utilization. J Telemed Telecare. 2018;24(7):473-81.

10 Yaeger KA, Shoirah H, Kellner CP, Johanna F, Mocco J, et al. Emerging technologies in optimizing pre-intervention workflow for acute stroke. Neurosurgery. 2019;85:S9-17.

11 Lumley HA, Flynn D, Shaw L, McClelland G, Ford GA, White PM, et al. A scoping review of pre-hospital technology to assist ambulance personnel with patient diagnosis or stratification during the emergency assessment of suspected stroke. BMC Emerg Med. 2020;20(1):30.

12 Ziegler V, Rashid A, Müller-Gorchs M, Kippnich U, Hiermann E, Kögerl C, et al. Mobile computing systems in preclinical care of stroke. Results of the Stroke Angel initiative within the BMBF project PerCoMed. Anaesthesist. 2008;57(7):677-85.

13 Ziegler V, Rashid A, Schaff M, Kippinch U, Griewing B. Quality management in acute stroke care: how to evaluate and improve the preclinical/clinical interface in stroke. Notarzt. 2012;28(6):237-45.

14 Soda H, Ziegler V, Shammas L, Griewing B, Kippinch U, Keidel M, et al. Telemedical prenotification in acute stroke treatment: experiences from the Stroke Angel initiative from 2004 until the present. Nervenarzt. 2017;88(2):120-9.

15 Pihlasviita S, Mattila OS, Ritvonen J, Sibolt G, Curtze S, Strbian D, et al. Diagnosing cerebral ischemia with door-to-thrombolysis times below 20 minutes. Neurology. 2018;91(6): e498-508.

16 Emberson J, Lees KR, Lyden P, Blackwell L, Albers G, Bluhmki E, et al. Effect of treatment delay, age, and stroke severity on the effects of intravenous thrombolysis with alteplase for acute ischaemic stroke: a meta-analysis of individual patient data from randomised trials. Lancet. 2014;384(9958):1929-35.

17 Andrew BY, Stack CM, Yang JP, Dodds JA. mStroke: "mobile stroke": improving acute stroke care with smartphone technology. J Stroke Cerebrovasc Dis. 2017;26(7):1449-56.

18 Meretoja A, Strbian D, Mustanoja S, Tatlisumak T, Lindsberg PJ, Kaste M. Reducing inhospital delay to 20 minutes in stroke thrombolysis. Neurology. 2012;79(4):306-13.

19 Bergrath S, Reich A, Rossaint R, Rörtgen D, Gerber J, Fischermann H, et al. Feasibility of prehospital teleconsultation in acute stroke-a pilot study in clinical routine. PLoS One. 2012;7(5):e36796.

20 Prabhakaran S, Lee J, O’Neill K. Regional learning collaboratives produce rapid and sustainable improvements in stroke thrombolysis times. Circ Cardiovasc Qual Outcomes. 2016;9(5):585-92.

21 Fayad P, Grotta JC. Evolving role of mobile stroke units within the prehospital stroke systems of care. Stroke. 2020 Jun 1;51(6):1637-8.

22 Lippman JM, Smith SN, McMurry TL, Sutton ZG, Gunnell BS, Cote J, et al. Mobile telestroke during ambulance transport is feasible in a rural EMS setting: the iTREAT study. Telemed J E Health. 2016;22(6):507-13.

23 Walter S, Kostopoulos P, Haass A, Lesmeister M, Grasu M, Grunwald I, et al. Point-of-care laboratory halves door-to-therapy-decision time in acute stroke. Ann Neurol. 2011;69(3): 581-6.

24 Xian Y, Xu H, Lytle B, Blevins J, Peterson ED, Hernandez AF, et al. Use of strategies to improve door-to-needle times with tissue-type plasminogen activator in acute ischemic stroke in clinical practice: findings from target: stroke. Circ Cardiovasc Qual Outcomes. 2017;10(1):e003227.10 